\title{
Pressure spectra in turbulent flows in the inertial and the dissipation ranges
}

Sipei Zhao and Eva ChengXiaojun Qiu and lan BurnettJacob Chia-chun Liu

Citation: J. Acoust. Soc. Am. 140, 4178 (2016); doi: 10.1121/1.4968881

View online: http://dx.doi.org/10.1121/1.4968881

View Table of Contents: http://asa.scitation.org/toc/jas/140/6

Published by the Acoustical Society of America

\section{Articles you may be interested in}

Noise control zone for a periodic ducted Helmholtz resonator system

J. Acoust. Soc. Am. 140, (2016); 10.1121/1.4968530

Acoustics from a rectangular supersonic nozzle exhausting over a flat surface

J. Acoust. Soc. Am. 140, (2016); 10.1121/1.4967158

Toward mechanistic understanding of the relationship between the sound absorption and the natural and resonant frequencies of porous media

J. Acoust. Soc. Am. 140, (2016); 10.1121/1.4968782

Scattering of turbulent-jet wavepackets by a swept trailing edge

J. Acoust. Soc. Am. 140, (2016); 10.1121/1.4971425 


\title{
Pressure spectra in turbulent flows in the inertial and the dissipation ranges
}

\author{
Sipei Zhao a) and Eva Cheng \\ School and Engineering, RMIT University, 124 La Trobe Street, Melbourne, Victoria 3000, Australia

\begin{abstract}
Xiaojun Qiu and lan Burnett
Centre for Audio, Acoustics and Vibration, Faculty of Engineering and IT, University of Technology Sydney, 15 Broadway, Ultimo, New South Wales 2007, Australia

Jacob Chia-chun Liu

Department of Water Resources and Environmental Engineering, Tamkang University, 151 Yingzhuan Road, Tamsui, Taipei 251, Taiwan
\end{abstract}

(Received 30 September 2016; revised 26 October 2016; accepted 12 November 2016; published online 7 December 2016)

\begin{abstract}
Based on existing studies that provide the pressure spectra in turbulent flows from the asymptotic pressure structure function in the inertial range, this paper extends the pressure spectrum to the dissipation range by proposing a pressure structure function model that incorporates both the inertial and dissipation ranges. Existing experiment results were used to validate the proposed pressure structure function model first, and then the obtained pressure spectrum was compared with the simulation and measurement data in the literature and the wind-induced noise measured outdoors. All comparisons demonstrate that the pressure spectrum obtained from the proposed pressure structure function model can be used to estimate the pressure spectra in both the inertial and dissipation ranges in turbulent flows with a sufficiently large Reynolds number. (C) 2016 Acoustical Society of America.
\end{abstract}

[http://dx.doi.org/10.1121/1.4968881]

[JFL]

Pages: 4178-4182

\section{INTRODUCTION}

Pressure fluctuations in turbulent flows have been widely studied in the past, and are usually denoted by the pressure structure function in the spatial domain. The pressure structure function describes the spatial relationship between pressures at two locations by $D_{p}(r)=\left\langle(p(x)-p(x+r))^{2}\right\rangle$, where $p(x)$ is the pressure at position $x, r$ is the separation distance between two spatial locations, and $\langle\cdot\rangle$ denotes the ensemble average. The pressure structure function can be related to the pressure correlation function $R_{p}(r)$ by $D_{p}(r)=2 R_{p}(0)-2 R_{p}(r)$, where $R_{p}(r)=\langle p(x) p(x+r)\rangle$ (Obukhov and Yaglom, 1951). The structure function was shown to be computed at a higher accuracy than the correlation function but with less data (Schulz-DuBois and Rehberg, 1981).

Batchelor (1951) derived the pressure correlation function from the Poisson Equation based on the assumption that the velocities at two spatial points are joint Gaussian. It was shown that the joint Gaussian assumption produces the same results as Heisenberg's assumption that the Fourier components of velocities are statistically independent (Batchelor, 1951). With this pressure correlation function, the pressure structure function varies as $r^{4 / 3}$ and hence the pressure spectrum varies as $k^{-7 / 3}$ ( $k$ is the wavenumber) within the inertial range, where the eddy motions are determined by inertial effects and the viscous effect is negligible (Batchelor, 1951; Hill and Wilczak, 1995). The joint Gaussian assumption is consistent with the experimental results indicating that the

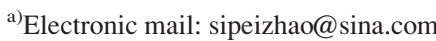

distribution of the velocity at one point is approximately normal (Townsend, 1947; Batchelor, 1951).

Instead of using the joint Gaussian assumption, Obukhov and Yaglom (1951) showed that $D_{p}(r)$ is proportional to $\varepsilon^{4 / 3} r^{4 / 3}$ from the dimensional analysis by assuming that the eddy motions are determined by the energy dissipation rate in the inertial range ( $\varepsilon$ is the energy dissipation rate). More than 40 years later, Hill and Wilczak (1995) developed a theoretical model to relate the pressure structure function to the fourth-order velocity structure functions, claiming the new theory to be valid for all Reynolds numbers and for all spatial separations and wavenumbers. Based on this theory, the $k^{-7 / 3}$ pressure spectrum in the inertial range was also obtained (Hill and Wilczak, 1995). In an alternative approach, George et al. (1984) developed spectral models for turbulent pressure fluctuations by directly applying the Fourier transform to the integral solution of the Poisson equation, showing that the turbulence-turbulence interaction decays as $k^{-7 / 3}$ in the inertial range.

Recently a series of outdoor wind noise measurements were carried out and compared to the above mentioned theories (Raspet et al., 2006; Raspet et al., 2008). Batchelor's theory (Batchelor, 1951) was utilized to predict the pressure spectrum in the inertial range from the measured velocity spectrum (Raspet et al., 2006). Based on the theoretical model proposed by George et al. (1984), Raspet et al. (2008) developed a spectral model extending to the low frequency region in the energy-containing range. The infrasonic wind noise spectrum was measured under a pine tree canopy and a deciduous tree canopy and it was found that the turbulenceshear interaction corresponds to the low frequency peak in 
the wind noise spectrum, while the turbulence-turbulence interaction pressure with the $-7 / 3$ power law dominates the higher frequency region in the inertial range (Raspet and Webster, 2015; Webster and Raspet, 2015).

The above-mentioned theories focus on the inertial range and assume that the $-7 / 3$ power law is also valid for the higher frequency region in the dissipation range. However, recent numerical simulations and experimental results showed that the pressure spectrum falls off much faster than the $-7 / 3$ power law, but no theory exists for predicting the pressure spectrum in the dissipation range (Gotoh and Fukayama, 2001; Tsuji and Ishihara, 2003). To describe the pressure spectrum in the higher frequency region, this paper proposes a pressure structure function model that incorporates both the inertial and dissipation ranges to obtain the pressure spectrum in the dissipation range. Existing simulation and measurement data from literature and our wind-induced noise measured outdoors are used to validate the proposed pressure structure function model and the obtained pressure spectrum.

\section{THEORY}

The pressure fluctuations in a viscous incompressible fluid are determined by the Poisson equation (Batchelor, 1951)

$$
\frac{1}{\rho} \nabla^{2} p=-\frac{\partial^{2} u_{i} u_{j}}{\partial x_{i} \partial x_{j}},
$$

where $\rho$ is the fluid density, $p$ is the pressure, and $u_{i}$ is the velocity along the $x_{i}$ direction. The pressure structure function is defined as (Hill and Wilczak, 1995)

$$
D_{p}(r)=\frac{1}{\rho^{2}}\left\langle(p(x)-p(x+r))^{2}\right\rangle,
$$

where $p(x)$ is the pressure at position $x, r$ is the separation distance between two spatial locations, and $\langle\cdot\rangle$ denotes the ensemble average.

Hill and Wilczak (1995) proposed a theoretical model to relate the pressure structure function to the fourth-order velocity structure function from Eq. (1), and showed that in the inertial range, the pressure structure function $D_{p}(r)$ can be simplified to a universal form

$$
D_{p}(r) \approx C_{p} \varepsilon^{4 / 3} r^{4 / 3}
$$

where $C_{p}$ is a constant. Equation (3) shows that in the inertial range, the pressure structure function is solely determined by the energy dissipation rate, and increases with the separation distance according to an exponent of $4 / 3$.

In the dissipation range, the pressure structure function for small separation distance $r$ should be approximated as (Obukhov and Yaglom, 1951; Hill and Wilczak, 1995)

$$
D_{p}(r) \approx \frac{1}{3} A r^{2}
$$

where $A=\int_{0}^{\infty} y^{-3} D_{1111}(y) d y$ is independent of $r$ (OuldRouis et al., 1996), $D_{1111}=\left\langle(u(x)-u(x+r))^{4}\right\rangle$ is the fourth order longitudinal velocity structure function and $u$ is the longitudinal velocity. Equation (4) shows that in the dissipation range, the square of the pressure difference at two spatial locations increases with the squared separation distance and increases at a faster rate than the inertial range.

The pressure spectrum can be calculated from the pressure structure function by (Lohse and Muller-Groeling, 1995)

$$
P(k)=-\frac{1}{2 \pi} \int_{0}^{\infty} D_{p}(r) \sin (k r) k r d r,
$$

where $k$ is the wavenumber. In existing research that focused on the inertial range, Eq. (3) was substituted into Eq. (5), and the pressure spectrum in the inertial range was obtained as (Hill and Wilczak, 1995)

$$
P(k) \approx 0.328 C_{p} \varepsilon^{4 / 3} k^{-7 / 3} .
$$

This inertial range pressure spectrum is consistent with the Kolmogorov's dimensional analysis and previous theoretical models (Batchelor, 1951; George et al., 1984), and has been validated by many simulation and experiment results when the Reynolds number is sufficiently large (Gotoh and Fukayama, 2001; Tsuji and Ishihara, 2003).

Unfortunately, the pressure spectrum in the dissipation range cannot be obtained by directly substituting Eq. (4) into Eq. (5), because the integral does not converge (Sirovich et al., 1994). To predict the pressure spectrum in a wider range, this paper proposes a pressure structure function model that incorporates both the inertial range [Eq. (3)] and the dissipation range [Eq. (4)] as

$$
D_{p}(r) \approx \frac{1}{3} \frac{A r^{2}}{\left[1+\left(r / r_{\mathrm{d}}\right)^{2}\right]^{1 / 3}},
$$

where $r_{\mathrm{d}}=\left(3 C_{p} \varepsilon^{4 / 3} / A\right)^{3 / 2}$ denotes the transition distance from the inertial range to the dissipation range, and can be obtained by equating Eq. (3) to Eq. (4). Previous results from experiments showed that the transition between the inertial range and dissipation range occurs at about $8.74 \eta<r_{\mathrm{d}}<11.25 \eta$ (Lohse and Muller-Groeling, 1996). For $r \gg r_{\mathrm{d}}$, Eq. (7) approaches Eq. (3) in the inertial range while for $r \ll r_{\mathrm{d}}$, Eq. (7) approaches Eq. (4) in the dissipation range.

By substituting Eq. (7) into Eq. (5), the pressure spectrum can be obtained (Lohse and Muller-Groeling, 1995)

$$
\begin{aligned}
P(k)= & \frac{2^{1 / 6} A r_{\mathrm{d}}{ }^{3}}{6 \sqrt{\pi} \Gamma\left(\frac{1}{3}\right)} \\
& \times\left[\frac{4}{3}\left(k r_{\mathrm{d}}\right)^{-1 / 6} K_{13 / 6}\left(k r_{\mathrm{d}}\right)+\left(k r_{\mathrm{d}}\right)^{5 / 6} K_{7 / 6}\left(k r_{\mathrm{d}}\right)\right],
\end{aligned}
$$

where $\Gamma()$ is the gamma function, and $K_{n}()$ is the second kind modified Bessel function of order $n$, which can be expanded in asymptotic forms as (Abranmowitz and Stegun, 1970; Mechel, 1966) 


$$
K_{n}(z) \approx \begin{cases}\frac{1}{2} \Gamma(n)\left(\frac{1}{2} z\right)^{-n}, & z \ll 1 \\ \left(\frac{\pi}{2}\right)^{1 / 2} z^{-1 / 2} e^{-z}, & z \gg 1 .\end{cases}
$$

Applying Eq. (9) to Eq. (8), the limiting form of the pressure spectrum can be obtained

$$
P(k) \approx \begin{cases}\frac{2^{7 / 3} A \Gamma\left(\frac{13}{6}\right) r_{\mathrm{d}}{ }^{3}}{9 \sqrt{\pi} \Gamma\left(\frac{1}{3}\right)}\left(k r_{\mathrm{d}}\right)^{-7 / 3}, & k r_{\mathrm{d}} \ll 1 \\ \frac{2^{1 / 6} A r_{\mathrm{d}}{ }^{3}}{12 \Gamma\left(\frac{1}{3}\right)}\left(k r_{\mathrm{d}}\right)^{1 / 3} e^{-k r_{\mathrm{d}},} & k r_{\mathrm{d}} \gg 1 .\end{cases}
$$

Equation (10) shows that the transition between the inertial range and the dissipation range occurs at $k=1 / r_{\mathrm{d}}$, and the inertial range and the dissipation range in the wavenumber space can be denoted as $k \ll 1 / r_{\mathrm{d}}$ and $k \gg 1 / r_{\mathrm{d}}$, respectively. In the inertial range $\left(k r_{\mathrm{d}} \ll 1\right)$, the pressure spectrum obtained from the proposed model shows the $-7 / 3$ power law, which is consistent with previous studies. In the dissipation range $\left(k r_{\mathrm{d}} \gg 1\right)$, the pressure spectrum falls off much faster than the $-7 / 3$ power law.

The limitation of the current work is that the proposed pressure structure function model is only valid for sufficiently large Reynolds number because the inertial range with $D_{p}(r) \sim r^{4 / 3}$ [or equivalently $P(k) \sim k^{-7 / 3}$ ] always exists in Eq. (7). This might not be true for the small Reynolds number turbulent flows because recent numerical simulation and experimental results showed that the inertial range with the $-7 / 3$ power law cannot be observed when the Reynolds number is small (Gotoh and Fukayama, 2001; Tsuji and Ishihara, 2003).

The numerical simulations by Gotoh and Fukayama (2001) showed that the $-7 / 3$ power law can be observed when the Taylor microscale Reynolds number is larger than 284 , while the experimental results in wind tunnels by Tsuji and Ishihara (2003) confirmed the $-7 / 3$ power law when the Taylor microscale Reynolds number is larger than 600 . Meldi and Sagaut (2013) argued that a Taylor microscale Reynolds number larger than $10^{4}$ is necessary to observe the $-7 / 3$ power law in the pressure spectrum. It is still not known whether there exists a value of the Reynolds number such that the $-7 / 3$ power law can be observed above this value.

It has been shown that the Reynolds number in atmospheric turbulence is usually large enough for the inertial range to be observed (Wyngaard, 2010). Therefore, the pressure spectrum obtained from the proposed pressure structure function model should be used for predicting the noise spectra induced from outdoor wind. In Sec. III, the proposed pressure structure function model and the obtained pressure spectrum will be validated with data obtained from existing literature and our wind-induced noise measured outdoors.

\section{VERIFICATIONS AND DISCUSSIONS}

\section{A. Verification with existing data}

Figure 1 compares the proposed pressure structure function model in Eq. (7) with existing experimental results from literature (Xu et al., 2007). However, the values of $\eta$ and $r_{\mathrm{d}}$ were not given in the literature with the experimental results; thus, the proposed pressure structure function model was fitted to the experimental results in Fig. 1 with $r_{\mathrm{d}}=10 \eta$. The experimental results in Fig. 1 are from the turbulent water flows between a pair of counter-rotating disks, which can be described by the incompressible viscous Navier-Stokes equations (Xu et al., 2007). The proposed model is based on the Poisson equation, which is also obtained from the incompressible viscous Navier-Stokes equations. Therefore, the experimental results can be used to validate the proposed model. Different from previous theories that assume that the inertial range $\left(D_{p}(r) \sim r^{4 / 3}\right)$ extends to an infinitely small separation distance, the proposed model in Eq. (7) shows a better agreement in Fig. 1 for small separation distances, where eddies in the dissipation range dominate the pressure structure function.

The pressure spectrum obtained from the proposed model in Eq. (8) is compared with the existing Direct Numerical Simulation (DNS) and experimental results in Fig. 2 (Gotoh and Fukayama, 2001; Tsuji and Ishihara, 2003). The experimental results in Fig. 2(b) were measured on the center line in the free jet from a small wind tunnel with a $40 \times 40 \mathrm{~mm}^{2}$ nozzle and a large wind tunnel with a $400 \times 700 \mathrm{~mm}^{2}$ nozzle. The Taylor microscale Reynolds number is in the range of $200 \leq R_{\lambda} \leq 1200$. The pressure fluctuations were measured with a standard $1 / 4$ in. condenser microphone for $R_{\lambda}<350$, and with a small piezoresistive transducer for $R_{\lambda}>350$ (Tsuji and Ishihara, 2003). The values of $\eta$ and $r_{\mathrm{d}}$ were not given in the literature with the simulation and experimental results, therefore the obtained pressure spectrum in Eq. (8) was fitted to the experimental results in Fig. 2 with $r_{\mathrm{d}}=10 \eta$.

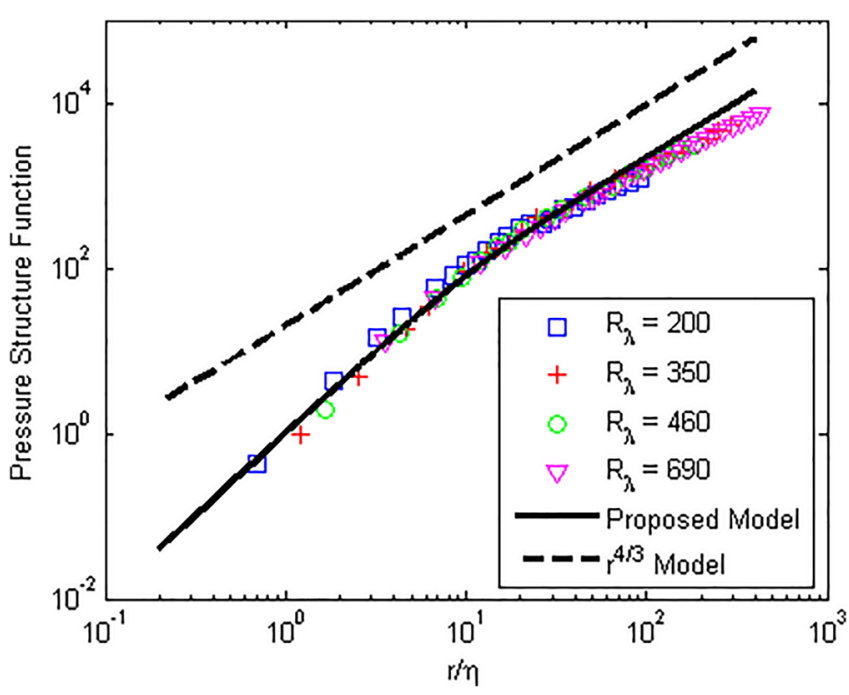

FIG. 1. (Color online) Comparison of the proposed pressure structure function model in Eq. (7) with the experimental results from $\mathrm{Xu}$ et al. (2007). The abscissa is normalized with the Kolmogorov scale $\eta$. 


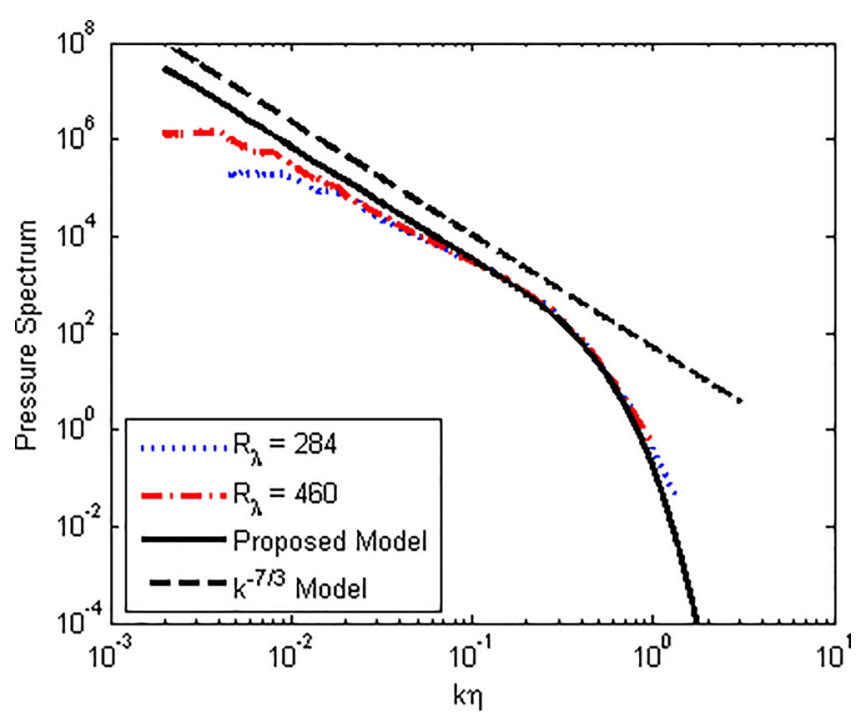

(a)

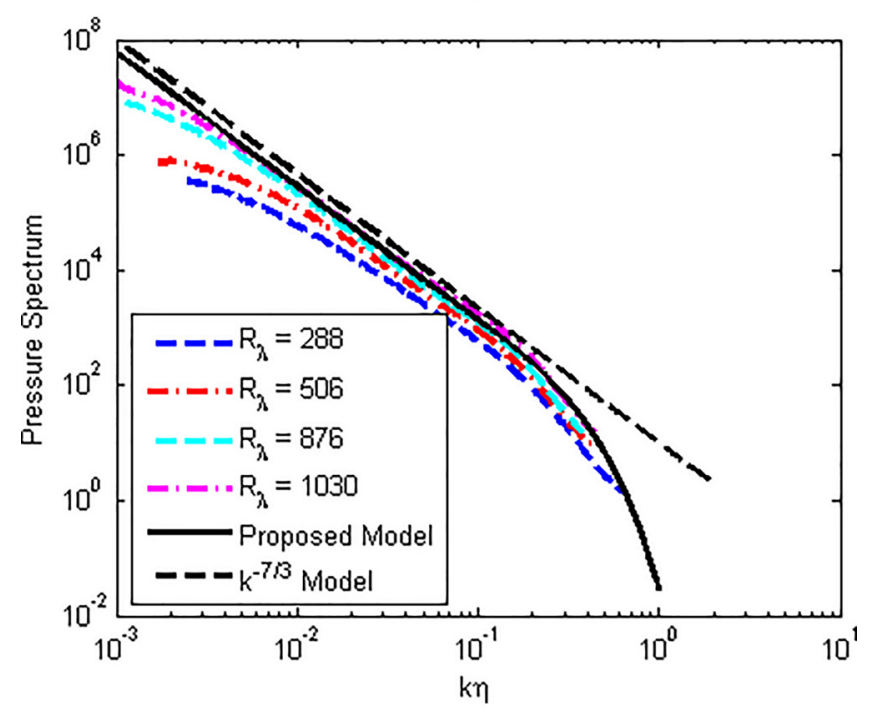

(b)

FIG. 2. (Color online) Comparison of the pressure spectra obtained from the proposed model in Eq. (8) with existing results, (a) DNS simulations by Gotoh and Fukayama (2001), and (b) wind tunnel experimental results by Tsuji and Ishihara (2003). The abscissa is normalized with the Kolmogorov scale $\eta$.

Figure 2 shows that the simulated and measured pressure spectra decay following the $-7 / 3$ power law in the inertial range at about $k \eta<0.2$, but begins to fall off rapidly at about $k \eta>0.2$, which deviates from the conventional $k^{-7 / 3}$ model. The pressure spectrum obtained from the proposed model in Eq. (8) is consistent with the simulation and experimental results in this rapid decay region in the dissipation range as well as the $-7 / 3$ power law in the inertial range, which cannot be predicted with the traditional asymptotic form pressure structure function.

\section{B. Outdoor wind noise measurements}

We measured wind-induced noise outdoors to further validate the pressure spectrum obtained from the proposed model. The experiments were carried out at dawn on October 8,2015 , at a car park in Taiwan, where there are no obstacles or reflective surfaces nearby. The wind speed was measured with a WindSonic Ultrasonic Wind Sensor anemometer (Gill Instruments LTD., Lymington, Hampshire, UK), and wind noise spectrum was measured with an unscreened RION NL32 1/2 in. Type UC-53A microphone (RION Co. Ltd., Tokyo, Japan). The anemometer and the microphone were mounted at the same height, at about $1.2 \mathrm{~m}$ above the ground, with a horizontal distance about $0.5 \mathrm{~m}$. The anemometer and the microphone were both connected to a RION DA-20 multi-channel processor and the wind speed and sound pressure level were stored per second.

The measurement results were originally in one-third octave bands, thus the narrowband spectra of the conventional $k^{-7 / 3}$ model and the pressure spectrum obtained from the proposed model in Eq. (8) were converted to one-third octave band spectra. The pressure spectrum obtained from the proposed model in Eq. (8) was fitted to the measurement results

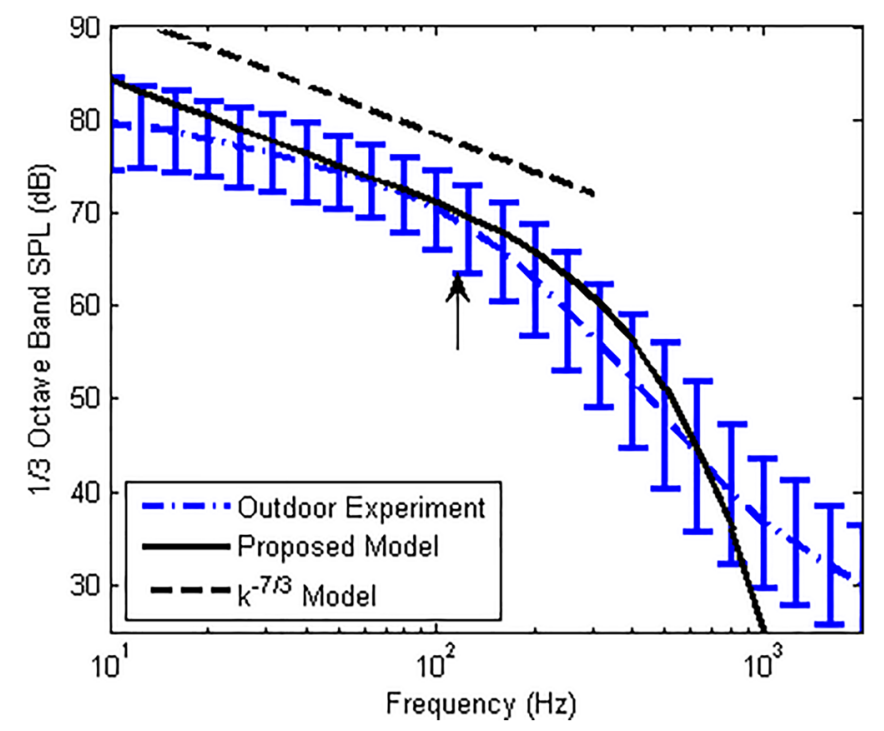

(a)

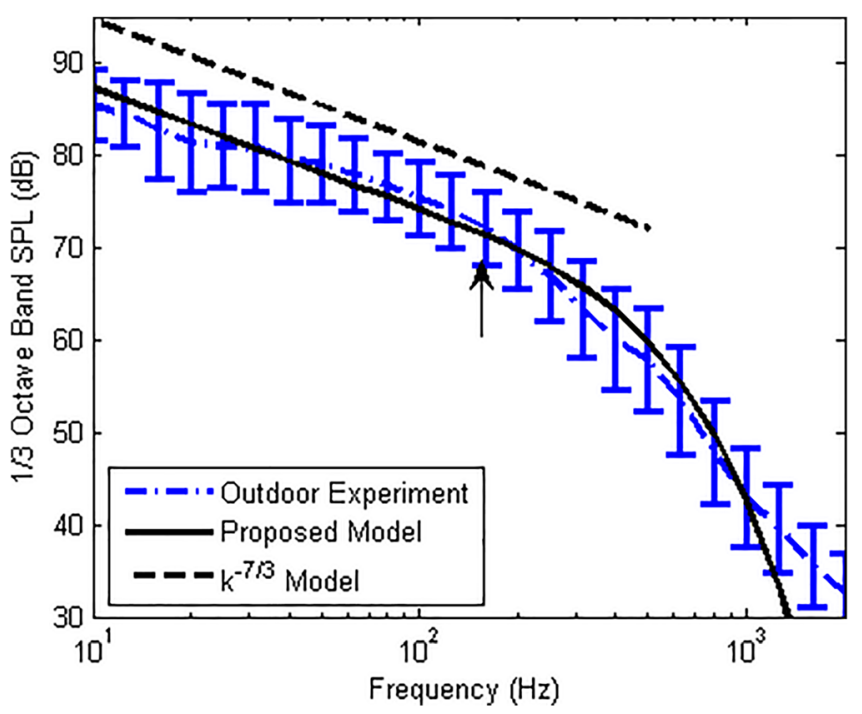

(b)

FIG. 3. (Color online) Comparison of the pressure spectra obtained from the proposed model in Eq. (8) with the outdoor experimental results at mean wind speeds of (a) $U=4.5 \mathrm{~m} / \mathrm{s}$ and (b) $U=5.5 \mathrm{~m} / \mathrm{s}$. The black arrows illustrate the transition from the inertial range and the dissipation range. 
from the unscreened microphone in Fig. 3 with $r_{\mathrm{d}}=6.8 \mathrm{~mm}$ and $r_{\mathrm{d}}=5.6 \mathrm{~mm}$ for the mean wind speeds of $U=4.5 \mathrm{~m} / \mathrm{s}$ and $U=5.5 \mathrm{~m} / \mathrm{s}$, respectively. The corresponding transition frequency between the inertial range and the dissipation range can be calculated with the Taylor's frozen turbulence hypothesis, i.e., $f_{\mathrm{d}}=U / 2 \pi r_{\mathrm{d}}$, as illustrated by the black arrows in Fig. 3. That is, $f_{\mathrm{d}}=105$ and $156 \mathrm{~Hz}$ correspond to the mean wind speeds of $U=4.5$ and $5.5 \mathrm{~m} / \mathrm{s}$, respectively. The measured wind-induced noise spectra in Fig. 3 were averaged around $U \pm 0.5 \mathrm{~m} / \mathrm{s}$ for the mean wind speed $U$ and the vertical bars indicate the standard deviation.

Figure 3 shows that in the inertial range to the left side of the black arrows, the outdoor wind noise spectra are consistent with the conventional $k^{-7 / 3}$ model; however, in the dissipation range to the right side of the black arrows the measured outdoor wind induced noise spectra fall off much more rapidly and deviate from the conventional $k^{-7 / 3}$ model. In contrast, the pressure spectra obtained from the proposed model in Eq. (8) agrees well with the measured outdoor wind noise spectra across the measured frequency range.

The outdoor wind-induced noise spectra in Fig. 3 were measured with an unscreened $1 / 2$ in. microphone, which might generate a wake behind and hence alter the wind-induced noise (Strasberg, 1988). However, it was shown that the dominant source of pressure fluctuations at the microphone outdoors is the intrinsic turbulence in the flow, rather than the fluctuating wake (Morgan and Raspet, 1992). Therefore the effect of the microphone on the outdoor wind-induced noise is not taken into account in Fig. 3.

\section{CONCLUSIONS}

This paper proposes a pressure structure function model that incorporates both the inertial range and the dissipation range to extend the pressure spectrum to the dissipation range in turbulent flows. The proposed pressure structure function model and the obtained pressure spectrum were found to be consistent with existing experimental and numerical simulation results. For further validation of the proposed model, outdoor wind-induced noise was measured and comparisons with the pressure spectrum obtained from the proposed pressure structure function model were found to match well in both the inertial range and the dissipation range. The limitation of the current work is that the proposed pressure structure function model is only valid for a sufficiently large Reynolds number when the inertial range exists. Future work will investigate the pressure structure function and the pressure spectrum with different Reynolds numbers.

\section{ACKNOWLEDGMENT}

This research was supported under Australian Research Council's Linkage Projects funding scheme (LP140100740).

Abramowitz, M., and Stegun, I. A. (1970). Handbook of Mathematical Functions (Dover Publications, Inc., New York), pp. 435-455.

Batchelor, G. K. (1951). "Pressure fluctuations in isotropic turbulence," Proc. Cambridge Philos. Soc. 47, 359-374.

George, W. K., Beuther, P. D., and Arndt, R. E. A. (1984). "Pressure spectra in turbulent free shear flows," J. Fluid Mech. 148, 155-191.

Gotoh, T., and Fukayama, D. (2001). "Pressure spectrum in homogeneous turbulence," Phys. Rev. Lett. 86(17), 3775-3778.

Hill, R. J., and Wilczak, J. M. (1995). "Pressure structure functions and spectra for locally isotropic turbulence," J. Fluid Mech. 296, 247-269.

Lohse, D., and Muller-Groeling, A. (1995). "Bottleneck effects in turbulence: Scaling phenomena in r versus p space,” Phys. Rev. Lett. 74(10), 1747-1750.

Lohse, D., and Muller-Groeling, A. (1996). "Anisotropy and scaling corrections in turbulence," Phys. Rev. E 54(1), 395-405.

Mechel, F. (1966). "Calculation of the modified Bessel functions of the second kind with complex argument," Math. Comp. 20(95), 407-412.

Meldi, M., and Sagaut, P. (2013). "Pressure statistics in self-similar freely decaying isotropic turbulence," J. Fluid Mech. 717(R2), 1-12.

Morgan, S., and Raspet, R. (1992). "Investigation of the mechanism of lowfrequency wind noise generation outdoors," J. Acoust. Soc. Am. 92(2), $1180-1183$.

Obukhov, A. M., and Yaglom, A. M. (1951). "The microstructure of turbulent flow," Prikl. Mat. Mekh. 15, 3-26. (Translation in NACA TM 1350, National Advisory Committee for Aeronautics, Washington, DC, June 1953).

Ould-Rouis, M., Antonia, R. A., Zhu, Y., and Anselmet, F. (1996). "Turbulent pressure structure function," Phys. Rev. Lett. 77(11), 2222-2224.

Raspet, R., and Webster, J. (2015). "Wind noise under a pine tree canopy," J. Acoust. Soc. Am. 137(2), 651-659.

Raspet, R., Webster, J., and Dillion, K. (2006). "Framework for wind noise studies," J. Acoust. Soc. Am. 119(2), 834-843.

Raspet, R., Yu, J., and Webster, J. (2008). "Low frequency wind noise contributions in measurement microphones," J. Acoust. Soc. Am. 123(3), $1260-1269$.

Schulz-DuBois, E. O., and Rehberg, I. (1981). "Structure function in lieu of correlation function," Appl. Phys. 24, 323-329.

Sirovich, L., Smith, L., and Yakhot, V. (1994). "Energy spectrum of homogeneous and isotropic turbulence in far dissipation range," Phys. Rev. Lett. 72(3), 344-347.

Strasberg, M. (1988). "Dimensional analysis of windscreen noise," J. Acoust. Soc. Am. 83(2), 544-548.

Townsend, A. A. (1947). "Measurements in the turbulent wake of a cylinder," Proc. R. Soc. A. 190(1023), 551-561.

Tsuji, Y., and Ishihara, T. (2003). "Similarity scaling of pressure fluctuation in turbulence," Phys. Rev. E. 68, 026309.

Webster, J., and Raspet, R. (2015). "Infrasonic wind noise under a deciduous tree canopy,” J. Acoust. Soc. Am. 137(5), 2670-2677.

Wyngaard, J. C. (2010). Turbulence in the Atmosphere (Cambridge University Press, Cambridge, UK), 393 pp.

$\mathrm{Xu}, \mathrm{H}$., Ouellette, T., Vincenzi, D., and Bodenschatz, E. (2007). "Experimental measurement of acceleration correlations and pressure structure functions in high Reynolds number turbulence," Phys. Rev. Lett. 99, 204501. 